\title{
Study on The Stability of Fiber Reinforced Thermoplastic
}

\author{
Vessel Liner \\ Liang $\mathrm{Wu}^{1, \mathrm{a}}$, Chenghong Duan ${ }^{1, \mathrm{~b},{ }^{,},{ }^{\prime} \text { Jinhao Huang }}{ }^{1}$ \\ ${ }^{1}$ CAE, Beijing University of Chemical Technology, Beijing, 100029, China \\ anickwuliang@gmail.com, ㅎduanchenghong@163.com
}

\begin{abstract}
Due to the particularity of the properties of the plastic liner material, the stability of the liner must be taken into account in the manufacture and inspection of the liner, especially the fiber winding. In this paper, the finite element buckling method is used to analyze the stability of the high density polyethylene liner, and the linear critical buckling load and the nonlinear critical buckling load are calculated respectively. The buckling morphology and load-displacement curve of the liner are obtained. Based on this analysis, the influence of the thickness of the liner and the aspect ratio on the two buckling loads is analyzed.
\end{abstract}

Key Words: Plastic liner, stability, buckling load, thickness, aspect ratio

\section{Introduction}

With the development of aerospace, nuclear industry and automobile industry, plastic liner composite material full-winding vessel have been paid more and more attention and application in foreign countries. However, there is no corresponding standard in the design and manufacture, and still lacking of application research of plastic liner full-winding vessel. So the item has a certain engineering significance.

In the fiber reinforced vessels, domestic and foreign scholars have done a certain research and exploration. Tomomasa Ohkubo et al [1] did the numerical simulation and calculation on the thermal impact of carbon fiber reinforced materials, through the finite difference method for carbon fiber heat transfer simulation. Falk K. Wittel et al [2] used the molecular dynamics tracking model to simulate the crack initiation and propagation process, and founded that the crack morphology was mainly affected by interface performance and lateral thickness. L.M.A.Cahill et al [3] founded that for the carbon fiber aerospace composites, fiber winding angle was the driving factor of crack propagation, and the specimen geometry, loading conditions and other factors had nothing to do. F. Rueda et al [4] through the three network model (TNM) simulation of HDPE liner pipe bending or extrusion collapse caused by the buckling of the corresponding strain rate and mechanical response being compared, put forward the corresponding design improvement method. JH Wang et al [5] analyzed the buckling equation of the non-circular cross-section composite lining, and obtained the buckling equation of the profiled inner lining. The critical pressure of the composite pipe lining under different environments was Analytical method. 
The study of the winding vessels shows that the pressure inside the vessel was mainly borne by the fiber wound layer, that was, fiber bearing, liner seal. Due to the material properties of the plastic liner, when the fiber winding or the temperature change is large, it was prone to external pressure instability. In order to understand the stability of thermoplastic plastic vessel liner more and help to determine the relevant parameters of the liner, this paper used finite element simulation method to study the stability of high density polyethylene liner.

\section{Finite element analysis}

\subsection{Analysis model}

In order to reflect the elastic and plastic properties of high density polyethylene materials, the calculation results are accurate and reliable. In this paper, only the material elasticity is considered in the process of eigenvalue buckling analysis, that is, only the elastic modulus and Poisson's ratio are defined. In the case of nonlinear buckling analysis, a bilinear constitutive model is used. The high density polyethylene material is shown in Fig. 2-1.

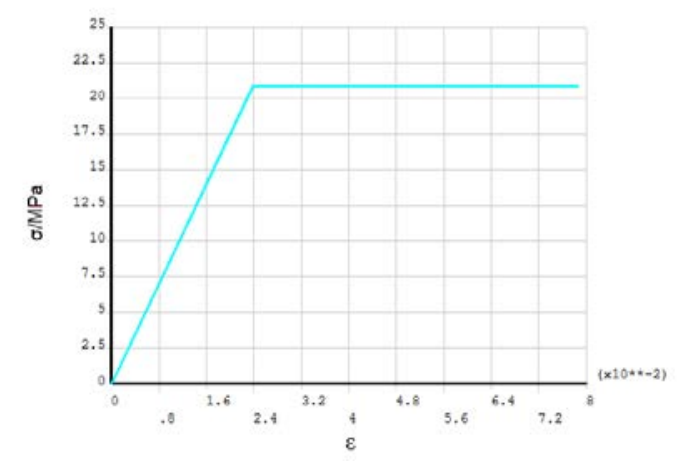

Fig.2-1 Stress-strain curve of double linear material

This paper uses ANSYS APDL for parametric modeling analysis and uses Solid 45 unit. The unit is defined by eight nodes, and each node has three degrees of freedom. The unit has the ability of plasticity, creep, stress strengthening, large deformation and large strain, which can save nonlinear calculation time while satisfying the requirement of high density polyethylene liner buckling. At the same time, the inner control of the liner is carried out with $10 \mathrm{mmx} 10 \mathrm{~mm}$ grid, and the discontinuous area of the opening and the head structure are divided into two parts, so as to ensure the definition of the initial defect of nonlinear calculation. The structure of the high density polyethylene liner is shown in Figure 2-2. The structural parameters are shown in Table 2-1. The finite element model is shown in Figure 2-3.

Table 2-1 Parameters of liner structure

\begin{tabular}{|c|c|c|c|c|c|c|}
\hline $\mathrm{L} / \mathrm{mm}$ & $\mathrm{L}_{\mathrm{h}} / \mathrm{mm}$ & $\mathrm{D}_{\mathrm{i}} / \mathrm{mm}$ & $\mathrm{t} / \mathrm{mm}$ & $\mathrm{D}_{10} / \mathrm{mm}$ & $\mathrm{D}_{1 \mathrm{i}} / \mathrm{mm}$ & $\mathrm{R} / \mathrm{mm}$ \\
\hline 760 & 600 & 300 & 2 & 19 & 15 & 10 \\
\hline
\end{tabular}




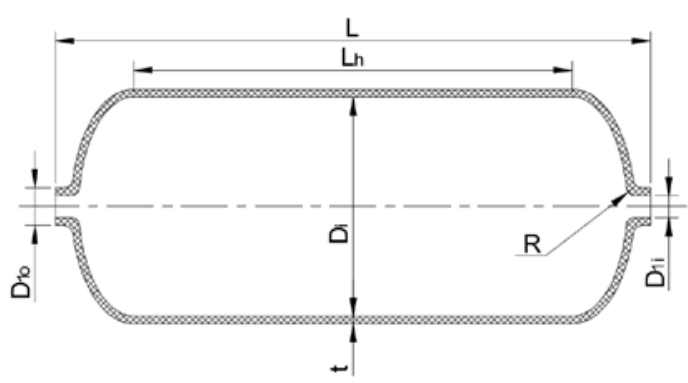

Fig.2-2 Structure of HDPE liner

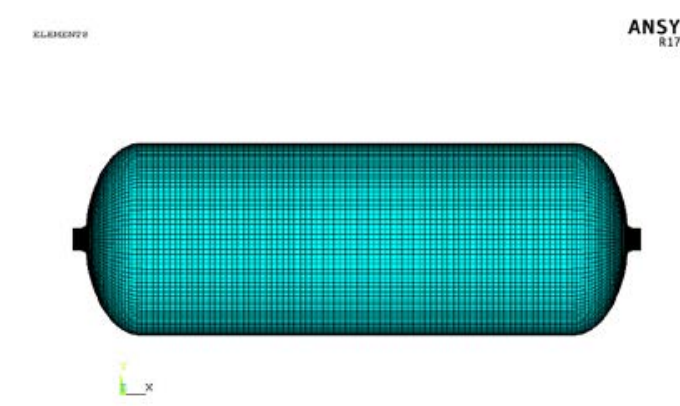

Fig.2-3 Finite element model of HDPE liner

In order to ensure the real reliability of buckling analysis, this paper determine the boundary conditions according to the actual force of high density polyethylene liner and the basic method of stability analysis. When the high-density polyethylene liner to withstand external pressure, one end restrain its axial displacement and rotation, the other end only restrain rotation. The outer surface of the liner apply the appropriate test external pressure and the corresponding nozzle load at the axial free end of.

\subsection{Analysis results}

\subsubsection{Buckling analysis results}

According to the above analysis method, this paper analyzes the characteristic buckling of high density polyethylene liner, and introduces the initial defect as the basis of nonlinear buckling analysis. The results are shown in Figures 2-4 and 2-5.

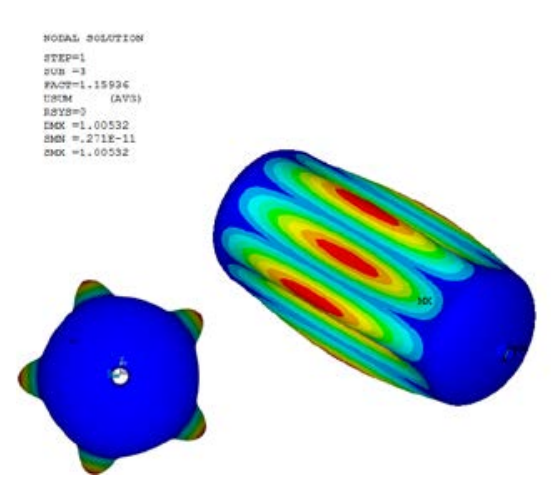

Fig.2-4 Deformation of buckling of bellows

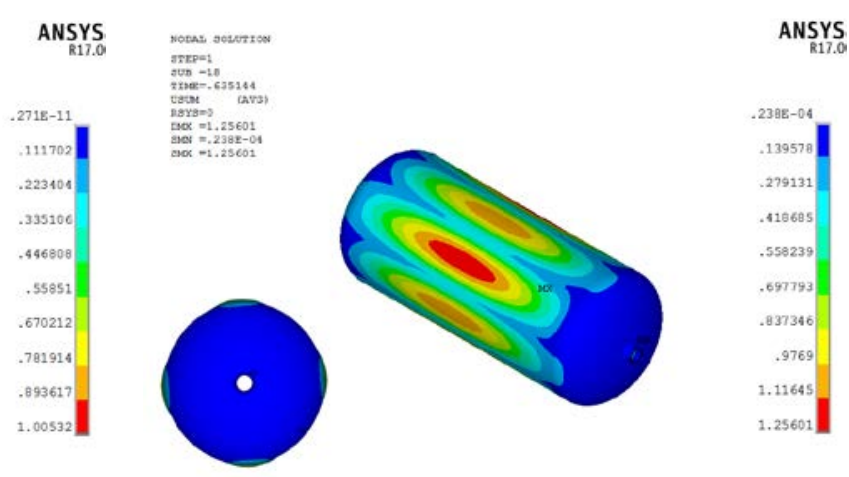

Fig.2-5 Deformation of nonlinear buckling of bellows

From the above deformation diagram, it can be seen that the liner has been unstable deformation. The main deformation is bulge and linear instability wave number are more than the number of nonlinear instability wave. From the calculation results, the results of the critical buckling load and the nonlinear critical buckling load are similar, and the results of the nonlinear buckling load are lower than the eigenvalue buckling load. The results of the nonlinear calculation are conserved compared with the linear results. 
The non-linear calculation of the displacement versus external pressure curve is shown in Figure 2-6. It can be seen from the figure, after the introduction of the initial defect, applying of external pressure, with the gradual increase in external pressure, the liner begin to shift in a wide range of basic linear relationship. When the pressure reaches the threshold, the pressure in the case of little change in the displacement begin to increase sharply and happen the liner instability. The critical pressure is a nonlinear critical buckling load.

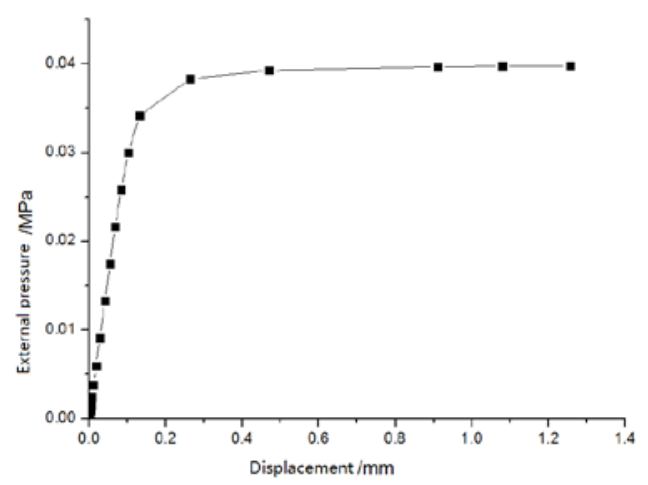

Fig.2-6 Curve changing with external pressure of liner deformation

\subsubsection{Effect of liner thickness on buckling load}

In order to understand the trend of the influence of the thickness of the liner on the buckling load, the buckling load of the intrinsic value in the thickness range of the commonly used liner is analyzed. Based on this, the nonlinear buckling load is calculated. The results are shown in Figure 2-7.

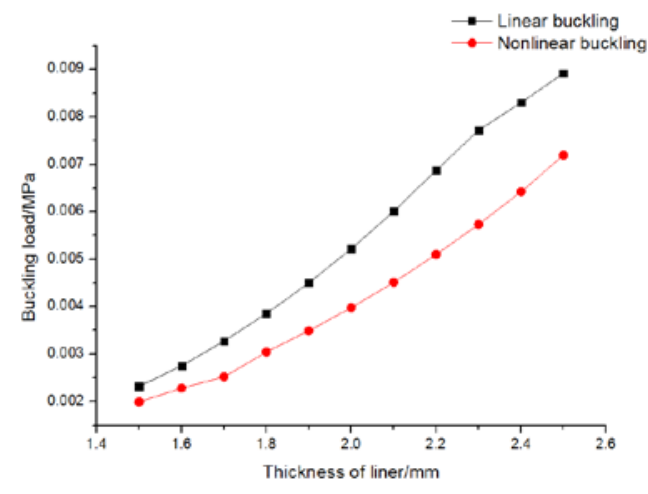

Fig.2-7 Curve changing with liner thickness of buckling loads

From Figure 2-7, it can be seen that the eigenvalue buckling load and the nonlinear buckling load of the high density polyethylene liner increase with the increase of the thickness. The trend of thickness variation agrees well with the theoretical analysis of external pressure stability, that is, with the increase of thickness, the overall resistance of the structure is enhanced. However, due to the thick liner will lead to a significant increase in the cost of the increase in the amount of winding layer, to enhance the weight of thermoplastic plastic vessels affect, so the need to be based on the actual needs of a reasonable choice. 


\subsubsection{The influence of the diameter ratio of the barrel section on the buckling load}

The critical length $\mathrm{L}_{\mathrm{cr}}$ is generally used to characterize the length of the cylinder, which is larger than the cylindrical shell of the size, which is the short cylinder, and the critical length of the cylindrical shell, the calculation of its critical pressure can be used when the long circle The cylinder formula can also use the short cylinder formula. The critical length is calculated as follows:

$$
L_{c r}=1.17 D_{0} \sqrt{\frac{D_{0}}{t}}
$$

The critical load of the long cylinder is expressed as:

$$
P_{c r}=\frac{2 E}{\left(1-\mu^{2}\right)}\left(\frac{t}{D}\right)^{3}
$$

For short cylinders, the critical pressure is calculated as follows:

$$
P_{c r}=\frac{E t}{R\left(1-\mu^{2}\right)}\left\{\frac{1-\mu^{2}}{\left(n^{2}-1\right)\left(1+\frac{n^{2} L^{2}}{\pi^{2} R^{2}}\right)^{2}}+\frac{t^{2}}{12 R^{2}}\left[\left(n^{2}-1\right)+\frac{2 n^{2}-1-\mu}{1+\frac{n^{2} L^{2}}{\pi^{2} R^{2}}}\right]\right\}
$$

According to the actual structural parameters of the enhanced thermoplastic vessel, it should be calculated according to the short cylinder. In order to better understand the relationship between the aspect ratio of the high density polyethylene liner and the critical pressure, the critical instability pressure of the different tube length is simulated. The calculated results are shown in Fig. 2-7.

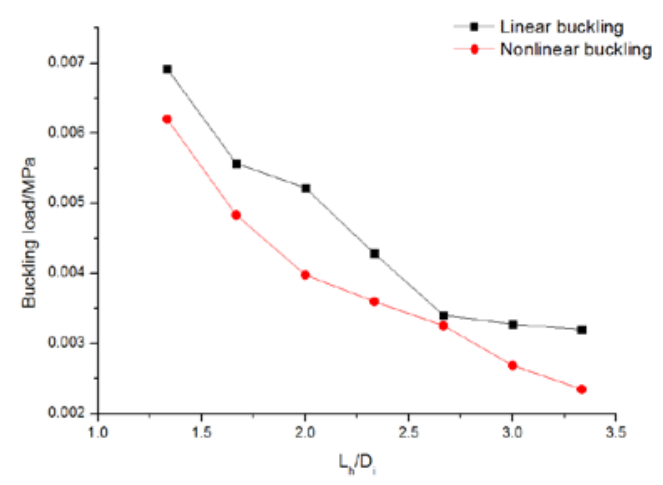

Fig.2-7 Curve changing with aspect ratio of liner deformation

The results show that with the increase of the aspect ratio, the buckling load decreases gradually, but the trend gradually slows down. As the aspect ratio increases, the overall stiffness of the vessel structure decreases and the external pressure stability is reduced which is prone to buckling. Therefore, in the design of enhanced thermoplastic vessel liner, in order to meet the requirements of the process parameters, it need to ensure that the lower aspect ratio. 


\section{Conclusion}

Based on the linear (eigenvalue) critical buckling load analysis and nonlinear critical buckling load analysis method, ANSYS APDL parameterization was used to analyze the stability of the high density polyethylene liner structure, and the basic form of the instability of the liner was obtained. On this basis, the influence of liner thickness and aspect ratio on critical buckling load was analyzed.

The results show that the critical buckling load of the high density polyethylene liner increases with the increase of the thickness of the liner, and the critical buckling load increases with the thickness of the inner layer. With the increase of the aspect ratio of the liner structure, the structural rigidity of the liner decreases, the resistance to external pressure decreases and the critical buckling load decreases. Therefore, in the vessel structure design, the stability of the liner should be calculated to ensure that the thickness of the liner and aspect ratio to meet the winding and thermal buckling stability requirements.

\section{References}

[1] Ohkubo T, Tsukamoto M, Sato Y. Numerical Simulation of Laser Beam Cutting of Carbon Fiber Reinforced Plastics[J]. Physics Procedia, 2014, 56: 1165-1170.

[2] Wittel F K, Schulte-Fischedick J, Kun F, et al. Discrete element simulation of transverse cracking during the pyrolysis of carbon fibre reinforced plastics to carbon/carbon composites[J]. Computational materials science, 2003, 28(1): 1-15.

[3] L.M.A. Cahill, S. Natarajan, S.P.A. Bordas, R.M. O’ Higgins, C.T. McCarthy. An experimental numerical investigation into the main driving force for crack propagation in uni-directional fibre-reinforced composite laminae [J]. Composite Structures,2014,107:119-130

[4] Rueda F, Torres J P, Machado M, et al. External pressure induced buckling collapse of high density polyethylene (HDPE) liners: FEM modeling and predictions[J]. Thin-Walled Structures, 2015, 96:56-63.

[5] Wang J H, Koizumi A, Yuan D J. Theoretical and numerical analyses of hydrostatic buckling of a noncircular composite liner with arched invert[J]. Thin-Walled Structures, 2016, 102:148-157. 